\title{
Regional Anesthesia for Pain Management After Orthopedic Procedures for Treatment of Lower Extremity Length Discrepancy
}

This article was published in the following Dove Press journal:

Journal of Pain Research

\author{
Mauricio Arce Villalobos' \\ Giorgio Veneziano (1D) \\ Christopher lobst ${ }^{3}$ \\ Rebecca Miller (D) ${ }^{2}$ \\ Ana Gabriela Walch (D) ${ }^{2}$ \\ Catherine Roth $\mathbb{I D}^{2}$ \\ Graciela Argote-Romero ${ }^{1,2}$ \\ David P Martin ${ }^{1,2}$ \\ Ralph J Beltran (D) ${ }^{1,2}$ \\ Joseph D Tobias (D) ${ }^{1,2}$ \\ 'Department of Anesthesiology \& Pain \\ Medicine, The Ohio State University \\ College of Medicine, Columbus, $\mathrm{OH}$, \\ USA; ${ }^{2}$ Department of Anesthesiology \& \\ Pain Medicine, Nationwide Children's \\ Hospital, Columbus, OH, USA; \\ ${ }^{3}$ Department of Orthopedic Surgery, \\ Nationwide Children's Hospital and The \\ Ohio State University College of \\ Medicine, Columbus, $\mathrm{OH}$, USA
}

\begin{abstract}
Introduction: The use of regional anesthesia techniques continues to expand in a wide variety of surgical procedures as the benefits and safety are increasingly appreciated. Limblengthening procedures are often associated with significant postoperative pain and high opioid requirements which may impact patient's recovery and increase risk of chronic pain and long-term opioid use.
\end{abstract}

Methods: The current study retrospectively reviews our experience utilizing a novel peripheral nerve catheter (PNC) protocol for postoperative pain management in patients undergoing elective limb-lengthening procedures. We measure total opioid consumption following $48 \mathrm{hrs}$ in the postoperative period between groups.

Results: A total of 70 patients were included from which 41 received general plus regional anesthesia (RA) and 29 were managed with general anesthesia alone (NORA). Postoperative pain needs were calculated as morphine equivalents (ME). There were no differences in the demographic characteristics between the groups. Over the first 48 postoperative hours, opioid use was $0.5 \mathrm{mg} / \mathrm{kg}$ ME (IQR 0.3, 0.9) in the RA group versus $1.7 \mathrm{mg} / \mathrm{kg}$ ME (IQR 1.1, 3.1) in the NORA group $(\mathrm{p}<0.001)$. Subgroup analysis between femoral lengthening and tibialfibular lengthening procedures demonstrated the same opioid-sparing effect favoring the RA group compared to the NORA group. Hospital length of stay was significantly shorter in the femoral lengthening RA group compared to NORA group (32 hrs [IQR 29, 35] versus $53 \mathrm{hrs}$ [IQR 33, 55], respectively). There was no significant difference in length of stay between the RA group and NORA group after tibial-fibular lengthening procedures.

Discussion: Regional anesthesia via continuous catheter infusions has a clinically significant opioid-sparing effect for postoperative pain management after limb-lengthening procedures and may facilitate earlier hospital discharge.

Keywords: peripheral nerve block, pediatric anesthesia, limb-lengthening procedures

\section{Introduction}

Over the last decade, accessible ultrasound technology, advances in clinical knowledge, and increased awareness of the benefits of regional anesthesia have expanded the use of regional anesthesia in pediatric orthopedic surgery. Despite this, the effectiveness of regional anesthesia in newer orthopedic techniques including limblengthening surgery remains unexplored. In the context of an ongoing opioid abuse epidemic, the search for effective non-opioid analgesic modalities for postoperative pain has become imperative. Unfortunately, postoperative consumption of prescribed opioids is associated with a high incidence of persistent opioid use in
Correspondence: Mauricio Arce Villalobos Department of Anesthesiology \& Pain Medicine, Nationwide Children's Hospital, 700 Children's Drive, Columbus, OH 43205, USA

$\mathrm{Tel}+|6| 4722-4200$

Fax $+1614722-4203$

Email Mauricio.ArceVillalobos@osumc.edu 
pediatric patients. ${ }^{1}$ To handle this challenge, the field of anesthesia has multiple therapeutic options to optimize acute pain control while minimizing opioid use. Regional anesthesia may be provided with a single-shot nerve block that provides analgesia for approximately 12 to $24 \mathrm{hrs}^{2}$ The use of peripheral nerve catheters with a continuous infusion of a local anesthetic agent provides even more prolonged analgesia and further limits the need for opioids for an extended postoperative period. ${ }^{3-6}$

Limb-lengthening procedures of the lower extremities may result in significant postoperative pain. ${ }^{7}$ Effective postoperative analgesia may improve the postoperative course, decrease the potential for adverse effects related to parenteral opioids, facilitate earlier recovery, and shorten hospital stays after these procedures. The application of regional anesthesia to this surgical population is complicated by their need for early physical therapy and the risk of intraoperative nerve injury and postoperative compartment syndrome. ${ }^{8}$ Based on these challenges, our regional anesthetic technique evolved. We began with single-shot peripheral nerve blockades, but realizing the drawbacks associated a short-term and dense block, soon transitioned into the insertion of peripheral nerve catheters (PNC). The PNCs were activated and infused according to a protocol with low concentration local anesthetic solution to provide prolonged analgesia while preventing motor blockade. We subsequently sought to characterize pain management after limb-lengthening procedures according to whether a regional anesthetic technique used.

\section{Methods}

This retrospective study was reviewed and approved by the Institutional Review Board of Nationwide Children's Hospital (study ID IRB18-00747), and was conducted in accordance with the Declaration of Helsinki. All subjects had given written informed consent to the surgical and anesthetic procedures. The need for additional informed consent was waived by the Institutional Review Board of Nationwide Children's Hospital because of the difficult nature of retrospectively obtaining written informed consent for research purposes. Patient data were used only for purposes of this study. Protected Health Information is not reported and we confirm patient data confidentiality. We retrospectively analyzed the electronic medical records of patients between 10 and 40 years of age with a body mass index (BMI) between 18 and $35 \mathrm{~kg} / \mathrm{m}^{2}$ who had undergone elective orthopedic surgery for treatment of lower extremity length discrepancy under general anesthesia (NORA) or general anesthesia plus regional anesthesia (RA). These patients were cared for using a multimodal analgesia regimen managed by the Acute Pain Service from the Department of Anesthesiology \& Pain Medicine at Nationwide Children's Hospital from December 2016 to January 2019.

The primary outcome was total postoperative opioid consumption over the first 48 postoperative hours. Opioid consumption was converted to oral morphine equivalents (ME) for overall comparison between groups. ${ }^{9}$ Secondary outcomes included a comparison of median pain scores using the Visual Analog Scale (VAS) in the first 48 hrs and length of stay following the procedure, discharge criteria included ability to actively participate in physical therapy and pain scores equal or less than 3 (VAS). Pain scores were collected in the post-anesthesia care unit (PACU) and at $2 \mathrm{hr}$ intervals during the first 48 postoperative hours. Intraoperative and postoperative opioid use, hospital length of stay, and postoperative pain scores were compared among the entire cohort according to receipt of regional anesthesia. To examine the effect of regional anesthesia among types of surgical procedures, the cohort was divided into two subgroups and the analysis was repeated. The two subgroups included patients undergoing femoral osteotomies with subsequent adjustable intramedullary nail insertion (PRECICE ${ }^{\circledR}$ ) and patients undergoing tibial-fibular osteotomies with subsequent placement of the Taylor Spatial Frame. Categorical variables were reported as counts with percentages and compared according to receipt of regional anesthesia using Chi-square tests. Continuous variables were reported as medians with interquartile ranges and compared using rank-sum tests. Data analysis was performed in Stata/IC 14.2 (College Station, StataCorp, LP) with two-tailed $\mathrm{P}<0.05$ considered statistically significant.

\section{Results}

The study cohort included a total of 70 patients, 37 females and 33 males with a median age of 14.5 years and a weight of 122 pounds (median value), data which is outlined in Table 1. General anesthesia was used alone in $29(42 \%)$ patients while general plus regional anesthesia was used in 41 (58\%) (Table 1). In both the RA and NORA groups, general anesthesia consisted of the intravenous induction of anesthesia with propofol (1-2 mg/kg) and fentanyl (1-2 $\mu \mathrm{g} / \mathrm{kg})$, followed by laryngeal mask airway placement or intravenous rocuronium $(0.6-1.2 \mathrm{mg} / \mathrm{kg})$ and endotracheal intubation according to the preference of the anesthesia provider. Anesthesia was maintained with desflurane or sevoflurane. Dexamethasone 
Table I Demographic Data of the Study Cohort $(\mathrm{N}=70)$

\begin{tabular}{|l|l|l|l|}
\hline $\begin{array}{l}\text { Demographic } \\
\text { Data }\end{array}$ & $\begin{array}{l}\text { Regional } \\
\text { Anesthesia } \\
\mathbf{( N = 4 1 )}\end{array}$ & $\begin{array}{l}\text { No Regional } \\
\text { Anesthesia } \\
(\mathbf{N}=29)\end{array}$ & P value \\
\hline Age (years) & $15(12,17)$ & $14(9,16)$ & 0.315 \\
Female & $23(56 \%)$ & $14(48 \%)$ & 0.518 \\
Height (inches) & $62(59,67)$ & $59(51,64)$ & 0.105 \\
Weight (pounds) & $119(98,150)$ & $125(7,189)$ & 0.976 \\
BMI (kg/m $\left.{ }^{2}\right)$ & $21.0(18,30)$ & $24.3(19,33)$ & 0.267 \\
\hline ASA Physical Status & & & \\
I & $10(24 \%)$ & $5(17 \%)$ & 0.691 \\
2 & $23(56 \%)$ & $20(69 \%)$ & \\
$\geq 3$ & $8(20 \%)$ & $4(14 \%)$ & \\
\hline
\end{tabular}

Note: The data are listed as median (IQR) or $\mathrm{N}(\%)$.

Abbreviations: IQR, interquartile range; N, number; BMI, body mass index; ASA American Society of Anesthesiologists.

$(0.1 \mathrm{mg} / \mathrm{kg}$, maximum of $4 \mathrm{mg})$, ondansetron $(0.1 \mathrm{mg} / \mathrm{kg}$, maximum $4 \mathrm{mg})$, intravenous acetaminophen $(10 \mathrm{mg} / \mathrm{kg}$, maximum $1 \mathrm{~g})$ and ketorolac $(0.5 \mathrm{mg} / \mathrm{kg}$, maximum $30 \mathrm{mg})$ were administered prior to the end of the case. Intravenous opioids were administered according to the clinical need as assessed by the autonomic response to surgical stimuli. As part of the postoperative analgesia regimen, all patients received acetaminophen (intravenous or oral, $10 \mathrm{mg} / \mathrm{kg}$ to a maximum of $1000 \mathrm{mg}$ every $4 \mathrm{hrs})$ plus ketorolac $(0.5 \mathrm{mg} / \mathrm{kg}$ to a maximum of $30 \mathrm{mg}$ every $6 \mathrm{hrs}$ ).

Patients undergoing femoral limb lengthening with adjustable intramedullary nail insertion (PRECICE ${ }^{\circledR}$ ) accounted for a total of 37 subjects with a mean age of 15 years: $11(30 \%)$ received general anesthesia while 26 $(70 \%)$ receive general plus regional anesthesia. At the beginning of the study period, the regional anesthetic techniques in this subgroup consisted of a postoperative femoral nerve catheter; however, the protocol evolved and was changed to a postoperative suprainguinal fascia iliaca catheter following the technique described by Hebbart et al. ${ }^{10}$ The catheter was inserted under ultrasound guidance with sterile technique below the inguinal ligament and adjacent to the femoral nerve (femoral nerve catheter) following lateral to medial direction. For the suprainguinal fascia iliaca catheter technique; the fascia iliaca was identified above the iliacus muscle; the needle was advanced under ultrasound guidance until it was positioned in between those structures and at the level of the deep circunflex iliac artery; then the space was opened using $0.9 \%$ normal saline and the catheter was threaded $4 \mathrm{~cm}$ past the needle tip . A test dose of epinephrine 1:200,000 $(0.5 \mu \mathrm{g} / \mathrm{kg}$ to a maximum dose of $15 \mu \mathrm{g})$ was administered to rule out intravascular catheter placement. A bolus dose of $0.2 \%$ ropivacaine $(0.1 \mathrm{~mL} / \mathrm{kg}$ of ideal body weight $)$ was administered after the surgeon performed a post-operative neurological exam in the post-anesthesia care unit (PACU) and an infusion of $0.1 \%$ ropivacaine was started at $0.1 \mathrm{~mL} /$ $\mathrm{kg} /$ hour.

In the tibial-fibular limb lengthening with placement of the Taylor Spatial Frame, there were a total of 33 patients with a mean age of 14 years. Eighteen patients (54\%) received general anesthesia and $15(46 \%)$ received general plus regional anesthesia. In this subgroup, the regional anesthesia technique consisted of the postoperative placement of adductor canal and sciatic nerve catheters under ultrasound guidance and sterile technique. After confirming proper position, a test dose of epinephrine 1:200,000 $(0.5 \mu \mathrm{g} / \mathrm{kg}$ to a maximum of dose of $15 \mu \mathrm{g})$ per catheter was administered to rule out intravascular placement. A bolus dose of $0.2 \%$ ropivacaine $(0.05 \mathrm{~mL} / \mathrm{kg}$ of ideal body weight) was administered per both catheters after proper bedside neurological exam was performed by the surgeon and an infusion of $0.1 \%$ ropivacaine was started at $0.05 \mathrm{~mL} / \mathrm{kg} /$ hour per both catheters. The decision related about timing on blousing PNC and starting infusion was based on our surgical team preference since they relate in their postoperative physical exam to rule out immediate neurological complications related to the surgical procedure which will not be interfered by local anesthetic at time of their exam.

In the overall cohort, there was a significant decrease in opioid consumption in the RA group compared to the NORA group (Table 2). Postoperative opioid use over the first $48 \mathrm{hrs}$ was $0.5 \mathrm{mg} / \mathrm{kg}$ of oral ME (IQR $0.3,0.9$ ) in RA group,

Table 2 Opioid Use, Length of Stay, and Pain Scores Among the Study Cohort $(\mathrm{N}=70)$

\begin{tabular}{|l|l|l|l|}
\hline $\begin{array}{l}\text { Outcome } \\
\text { Parameter }\end{array}$ & $\begin{array}{l}\text { Regional } \\
\text { Anesthesia } \\
\mathbf{( N = 4 1 )}\end{array}$ & $\begin{array}{l}\text { No Regional } \\
\text { Anesthesia } \\
(\mathbf{N}=\mathbf{2 9})\end{array}$ & P value \\
\hline $\begin{array}{l}\text { Intraoperative ME } \\
\text { (mg/kg) }\end{array}$ & $0.9(0.6,1.0)$ & $0.7(0.5,1.0)$ & 0.698 \\
\hline $\begin{array}{l}\text { First 48 postoperative } \\
\text { hours ME (mg/kg) }\end{array}$ & $0.5(0.3,0.9)$ & $1.7(\mathrm{I} . \mathrm{I}, 3.1)$ & $<0.00 \mathrm{I}$ \\
\hline Hospital LOS (hours) & $32(30,54)$ & $36(32,57)$ & 0.109 \\
\hline $\begin{array}{l}\text { Pain scores (first } 48 \\
\text { postoperative hours) }\end{array}$ & $2(0,3)$ & $2(1,3)$ & 0.415 \\
\hline
\end{tabular}

Note: The data are listed as median (IQR) or N (\%).

Abbreviations: $M E$, morphine equivalents; IQR, interquartile range; $N$, number; LOS, length of stay. 
Table 3 Opioid Use, Length of Stay, and Pain Scores Among Femoral Osteoplasty Patients $(\mathrm{N}=37)$

\begin{tabular}{|l|l|l|l|}
\hline $\begin{array}{l}\text { Outcome } \\
\text { Parameter }\end{array}$ & $\begin{array}{l}\text { Regional } \\
\text { Anesthesia } \\
\mathbf{( N = 2 6 )}\end{array}$ & $\begin{array}{l}\text { No Regional } \\
\text { Anesthesia } \\
\mathbf{( N = 1 1 )}\end{array}$ & P value \\
\hline $\begin{array}{l}\text { Intraoperative ME } \\
\text { (mg/kg) }\end{array}$ & $0.9(0.6,1.0)$ & $0.6(0.5,1.2)$ & 0.642 \\
\hline $\begin{array}{l}\text { First 48 postoperative } \\
\text { hours ME (mg/kg) }\end{array}$ & $0.4(0.2,0.9)$ & $2.1(0.9,3.1)$ & 0.006 \\
\hline Hospital LOS (hours) & $32(29,35)$ & $53(33,55)$ & 0.026 \\
\hline $\begin{array}{l}\text { Pain scores (first } 48 \\
\text { postoperative hours) }\end{array}$ & $2(0,3)$ & $2(0,2)$ & 0.460 \\
\hline
\end{tabular}

Note: The data are listed as median (IQR) or N (\%).

Abbreviations: $M E$, morphine equivalents; IQR, interquartile range; $N$, number; LOS, length of stay.

Table 4 Opioid Use, Length of Stay, and Pain Scores Among Tibial-Fibular Osteoplasty Patients (N=33)

\begin{tabular}{|l|l|l|l|}
\hline $\begin{array}{l}\text { Outcome } \\
\text { Parameter }\end{array}$ & $\begin{array}{l}\text { Regional } \\
\text { Anesthesia } \\
\mathbf{( N = 1 5 )}\end{array}$ & $\begin{array}{l}\text { No Regional } \\
\text { Anesthesia } \\
\mathbf{( N = 1 8 )}\end{array}$ & P value \\
\hline $\begin{array}{l}\text { Intraoperative ME } \\
\text { (mg/kg) }\end{array}$ & $0.9(0.5,1.1)$ & $0.9(0.5,1.0)$ & 0.664 \\
\hline $\begin{array}{l}\text { First } 48 \text { postoperative } \\
\text { hours ME (mg/kg) }\end{array}$ & $0.7(0.3,1.1)$ & $1.4(1.1,3.3)$ & 0.002 \\
\hline Hospital LOS (hours) & $49(32,57)$ & $34(31,59)$ & 0.789 \\
\hline Pain scores (48 hrs) & $0.5(0,3.5)$ & $2(1,5)$ & 0.190 \\
\hline
\end{tabular}

Note: The data are listed as median (IQR) or $\mathrm{N}(\%)$.

Abbreviations: $M E$, morphine equivalents; IQR, interquartile range; $N$, number; LOS, length of stay.

compared to $1.7 \mathrm{mg} / \mathrm{kg}$ of ME (IQR 1.1, 3.1) in the NORA group ( $\mathrm{p}<0.001$ ). The same outcome was observed in the two subgroups (Tables 3 and 4). Forty-eight-hour postoperative opioid consumption in the femoral limb-lengthening group receiving general plus regional anesthesia was $0.4 \mathrm{mg} / \mathrm{kg} \mathrm{ME}$ (IQR $0.2,0.9$ ) vs $2.1 \mathrm{mg} / \mathrm{kg}$ ME (IQR $0.9,3.1$ ) in the general anesthesia only subgroup $(\mathrm{p}=0.006)$. In the tibial-fibular lengthening subgroup, the general plus regional anesthesia patients required $0.7 \mathrm{mg} / \mathrm{kg} \operatorname{ME}(0.3,1.1)$ compared to the general anesthesia only subgroup $1.4 \mathrm{mg} / \mathrm{kg} \mathrm{ME}$ (IQR1.1, 3.3) $(\mathrm{p}=0.002)$ after 48 postoperative hours.

In the overall cohort, median length of stay (LOS) in the RA group was $32 \mathrm{hrs}$ (IQR 30, 54) versus $36 \mathrm{hrs} \mathrm{(IQR} \mathrm{32,} \mathrm{57)}$ in the NORA group (IQR 32, 57, p=0.109). In subgroup analysis, patients undergoing tibial-fibular lengthening had a similar hospital LOS whether they received regional anesthesia or general anesthesia only (49 hrs [IQR 32, 57] versus 34 hrs [IQR 31, 59], respectively). However, in the femoral lengthening subgroup, there was a significantly shorter hospital LOS in the regional anesthesia group (32 hrs [IQR 29, 35]) compared to the general anesthesia only group (53 hrs [IQR 33, 55]) $(\mathrm{p}=0.026)$. In the first 48 postoperative hours, there was no difference in the median pain scores in the RA group (2 [IQR 0,3]) and the NORA group $(2[\mathrm{IQR} 1,3])$. The same result was observed in pain scores in both procedure type subgroups.

\section{Discussion}

In this report, we describe the implementation of a novel regional anesthesia protocol that was able to significantly decrease postoperative opioid consumption while providing excellent analgesia following lower limb-lengthening procedures. Our results demonstrate the opioid-sparing effect could be maintained through the first 48 postoperative hours. Opioid requirements were 3.4 times higher in the NORA group compared to the RA group. This benefit was noted in both surgical subgroups, with femoral lengthening and tibial-fibular lengthening subgroups requiring approximately 5 and 2 times more opioid in the NORA group compared to the RA group, respectively. The pain associated with limb-lengthening procedures persists beyond the temporal capacity of a single-shot nerve block. The use of continuous local anesthetic infusions via PNCs supplied the necessary extended duration of analgesia beyond the typical 12 to $24 \mathrm{hr}$ duration expected with a single-shot nerve blockade.

In addition, the use of low concentration local anesthetic solution at conservative infusion rates avoided potential pitfalls of regional anesthesia including dense sensory and motor block which may prevent participation in physical therapy, mask nerve injury, and delay detection of compartment syndrome. All of the patients were able to effectively engage in physical therapy and do weightbearing exercises in the first $24 \mathrm{hrs}$ after the surgical procedure. Muscle weakness impeding recovery did not occur and their overall pain management was satisfactory without a difference noted between the groups, with overall pain scores generally below 3 .

The prevalence of moderate to severe pain in hospitalized children has been estimated to be $27 \%$ with patients admitted to surgical services accounting for $44 \%$ of this total patient population. ${ }^{11}$ One of the most important risk factors for developing chronic pain among pediatric surgical population is the presence of severe acute postoperative pain. ${ }^{12}$ The incidence of chronic postoperative pain 
may be as high as $13 \%$ with many of these patients undergoing orthopedic surgical procedures. ${ }^{13}$ Acute pain that is not adequately controlled appears more likely to evolve into chronic pain, which in addition to affecting the patient's quality of life, has significant health care and societal costs.

Recent surgical advances have allowed improved treatment of limb length discrepancy and angular deformities. Applying the Ilizarov principles, patients suffering from this pathology can now seek surgical correction involving osteotomies and osteoplasty with the subsequent application of controlled distraction devices promoting "distraction osteogenesis". ${ }^{14}$ In order for this intervention to succeed, controlled forces of movement and weight-bearing need to the applied to the healing bone. Adequate postoperative analgesia in this surgical population is required for the patients to engage in physical therapy which is mandatory and impacts in a positive manner the outcome of this procedure. $^{7,14}$

Regional anesthesia has been demonstrated to be safe in pediatric patients. ${ }^{15}$ The risk of transient neurological deficit is $2.4 / 10,000$ with a permanent neurological deficit incidence of $0.4 / 10,000 .{ }^{16}$ Nerve injury is a severe complication that may result from limb-lengthening procedures. The prevalence of this complication has been reported to be approximately $9.3 \%$ with $16 \%$ being present in the immediate postoperative period. ${ }^{17}$ The other $84 \%$ developed during the distraction phase. The local anesthetic agents used in regional anesthesia, block motor and sensory fibers and may mask symptoms related to nerve injury. In limb-lengthening procedures, which have a relatively high potential for nerve injury, potentially masking signs of nerve compromise is problematic. In our institution, we were able to develop a protocol in which patients received postoperative analgesia with regional anesthesia without masking symptoms of nerve injury or impeding patients engagement in physical therapy due to muscle weakness. The catheters were dosed only after the initial postoperative physical examination by the attending orthopedic surgeon. Additionally, lower doses and concentrations of the anesthetic agent were used (ropivacaine $0.1 \%$ ) at conservative infusion rates to limit the chances of motor blockade.

During the study, our protocol evolved in the femoral osteotomies subgroup. Initially, a femoral catheter alone was used as our main regional anesthesia technique. Although femoral nerve coverage was sufficient with this approach, lateral incisions in the thigh region were not adequately cover since the lateral femoral cutaneous nerve was spared with this approach. With the suprainguinal fascia iliaca technique, both the femoral and lateral femoral cutaneous nerves are covered in a consistent fashion. The majority of patients were discharged to home with the PNC remaining in place and an ambulatory pump continuing to infuse the local anesthetic. The PNC was typically removed on postoperative day 5 in these patients. Although these patients were followed with phone calls on a daily basis, no data was collected regarding opioid consumption beyond the first postoperative $48 \mathrm{hrs}$.

As expected, there were no differences in intraoperative opioid requirements between the groups due to the fact that the regional anesthesia techniques were performed after the surgical procedure. After completion of surgery but prior to leaving the operating room, our regional anesthesia team placed the peripheral nerve catheters without administering local anesthetic agents in order to avoid masking any neurological deficits that may have occurred because of the surgical procedure. In the recovery room, patients in the regional group reporting discomfort prior to the surgeon performing a neurologic exam of the involved extremity were treated with intravenous opioids. Our preliminary data demonstrate the potential postoperative benefits including decreased opioid requirements with maintenance of adequate analgesia and faster hospital discharge in femoral osteoplasty patients.

\section{Disclosure}

The abstract of this paper was presented at the 2019 American Society of Anesthesiologists annual meeting (2019 Orlando, FL.) as an abstract presentation with interim findings. Dr Christopher Iobst reports personal fees from NuVasive, personal fees from Orthofix, personal fees from Smith and Nephew, outside the submitted work. The authors report no other conflicts of interest in this work.

\section{References}

1. Harbaugh CM, Lee JS, Hu HM, et al. Persistent opioid use among pediatric patients after surgery. Pediatrics. In press 2018.

2. Ilfeld BM. Continuous peripheral nerve blocks: a review of the published evidence. Anesth Analg. 2011;113:904-925. doi:10.1213/ ANE.0b013e3182285e01

3. Gable A, Burrier C, Stevens J, et al. Home peripheral nerve catheters: the first 24 months of experience at a children's hospital. J Pain Res. 2016;9:1067-1072. doi:10.2147/JPR.S110947

4. Williams BA, Kentor ML, Vogt MT, et al. Reduction of verbal pain scores after anterior cruciate ligament reconstruction with 2-day continuous femoral nerve block. Anesthesiology. 2006;104:315-327. doi:10.1097/00000542-200602000-00018 
5. Ilfeld BM, Smith DW, Enneking FK. Continuous regional anesthesia following ambulatory pediatric orthopedic surgery. Am J Orthop. 2004;33:405-408.

6. Schloss B, Bhalla T, Klingele K, Phillips D, Prestwich B, Tobias JD. A retrospective review of femoral nerve block for postoperative analgesia after knee surgery in the pediatric population. $J$ Pediatr Orthop. 2014;34:459-461. doi:10.1097/BPO.0000000000000113

7. Young N, Bell DF, Anthony A. Pediatric pain patterns during Ilizarov treatment of limb length discrepancy and angular deformity. J Pediatr Orthop. 1994;14:352-357. doi:10.1097/01241398-199405000-00015

8. Fragomen AT. Transitioning to an intramedullary lengthening and compression nail. J Orthop Trauma. 2017;1:S7-S13. doi:10.1097/ BOT.0000000000000842

9. Gammaitoni AR, Fine P, Alvarez N, McPherson ML, Bergmark S. Clinical application of opioid equianalgesic data. Clin J Pain. 2003;19:286-297. doi:10.1097/00002508-200309000-00002

10. Hebbard P, Ivanusic J, Sha S. Ultrasound-guided supra-inguinal fascia iliaca block: a cadaveric evaluation of a novel approach. Anaesthesia. 2011;66:300-305. doi:10.1111/anae.2011.66.issue-4

11. Groenewald CB, Rabitts JA, Schroeder DR, Harrison TE. Prevalence of moderate-severe pain in hospitalized children. Pediatr Anesth. 2012;22:661-668. doi:10.1111/j.1460-9592.2012.03807.x
12. Nikolajsen L, Brix LD. Chronic pain after surgery in children. Curr Opin Anesthesiol. 2014;27:507-512. doi:10.1097/ACO.000000000 0000110

13. Fortier MA, Chou J, Maurer EL, Kain ZN. Acute to chronic postoperative pain in children: preliminary findings. J Pediatr Surg. 2011;46:1700-1705. doi:10.1016/j.jpedsurg.2011.03.074

14. Ledford C, Garrigues GE, Fitch RD. Ilizarov: the man, the myth, the method: an orthopedic inspiration. Duke Ortho J. 2013;3:104-107. doi:10.5005/jp-journals-10017-1039

15. Muhly W, Gurnaney H, Hosalkar H, Kraemer F, Davidson R, Ganesh A. Continuous peripheral infusion after lower extremity osteotomies in children: a feasibility and safety analysis. $\mathrm{Br}$ $J$ Anaesth. 2013;110(5):851-852. doi:10.1093/bja/aet100

16. Walker BJ, Long JB, Sathyamoorthy $\mathrm{M}$, et al. Complications in pediatric regional anesthesia, An analysis of more than 100,000 blocks for the pediatric regional anesthesia network. Anesthesiology. 2018;129:721-732. doi:10.1097/ALN.0000000000002372

17. Nogueira MP, Paley D, Bhave A, Nocente C, Herzenberg JE. Nerve lesions associated with limb-lengthening. J Bone Joint Surg Am. 2003;85A:1502-1510. doi:10.2106/00004623-200308000-00011

\section{Publish your work in this journal}

The Journal of Pain Research is an international, peer reviewed, open access, online journal that welcomes laboratory and clinical findings in the fields of pain research and the prevention and management of pain. Original research, reviews, symposium reports, hypothesis formation and commentaries are all considered for publication. The manuscript management system is completely online and includes a very quick and fair peer-review system, which is all easy to use. Visit http:// www.dovepress.com/testimonials.php to read real quotes from published authors. 